\title{
Dependable Medical Network Based on 2G and 3G Cellular Mobile Standards with External Channel Code in Concatenated Code Structure
}

\author{
E. Ahmed and R. Kohno
}

\begin{abstract}
This paper come as a proposal to enhance the error control coding in $2^{\text {nd }}$ generation $(2 G)$ and $3^{\text {rd }}$ generation (3G) for mobile communication to be capable to transmit the medical data by high level of reliability. The proposal bases on add extra code (convolution code) to the cellular mobile standard codes as concatenated channel code structure. The proposal transmits the medical data through this new channel that adopted as Medical Network Channel Code (MNCC) through mobile cellular networks. By this proposal we optimize the Bit Error Rate (BER) equal to the level of $10^{-5}$ with low SNR level that is a requirement needed for transmission medical data. The performance of the proposal MNCC compared based on 2G and 3G cellular networks. The performance of the MNCC has been proven and investigated using computer simulations under different channels condition such as, Additive White Gaussian Noise (AWGN) and Rayleigh Fading.
\end{abstract}

Index Terms-Cellular networks, error correction code, concatenated codes

\section{INTRODUCTION}

We are looking to the wireless and mobile networks and wide coverage provided by cellular networks, and we are thanks the technologies that brought possibilities in the field of medical transmission. In general, the design of the mobile telemedicine and tele-monitoring systems based on cellular networks was addressed in [1], [2]. Transmission of physiological signals, such as, electrocardiogram (ECG) and blood oxygen saturation ( $\mathrm{SpO} 2)$ by telecommunication means called Tele-monitoring, which is one of the telecommunication techniques that access delivery to healthcare services for people [2]. Consequently in this paper we are focusing on building a system that can serve a transmission of medical data robustly base on the cellular networks technologies. The main parts in the wireless and mobile transmission systems are the transmitter, transmission channel and the receiver. The transmission channel always affect by the noisy environment that makes it usually difficult to model. In the receiver side, error detection and correction techniques can be applied to provide better reception quality. The essential part of mobile communication is to ensure that the receiver can correctly interpret the transmitted signals. Errors control coding plays an important role for digital signals to become more reliable in a noisy communications environment [3].

Manuscript received October 25, 2013; revised March 20, 2014

The authors are with Division of Physics, Electrical and Computer Engineering, Faculty of Engineering, Yokohama National University, Yokohama, Japan (e-mail: sola@kohnolab.dnj.ynu.ac.jp, kohno@ynu.ac.jp).
There are many error correction techniques each one suitable with specific noisy environment such as random error or burst error or multipath distortion or channel effects or soon. Among of those techniques, there is a concatenation error correction codes technique that combines number of codes to work together. The idea of constructing high performance coding scheme by the concatenation of simpler codes is proposed by Forney [4] and the structure of such concatenation codes comprises of reed Solomon code as outer code and convolution code as inner code was addressed in [3].

The structure of the MNCC over Global System for Mobile communication (GSM) was detailed in [5]. Therefore we can say without any other reliable network infrastructure, GSM can be applied for tele-monitoring applications, where high mobility and low cost are necessary. The GSM channel coding scheme for traffic channel mainly work by convolution coding and the modulation techniques is Gaussian Minimum Shift Keying (GMSK). Universal Mobile Telecommunications System (UMTS) is the main standard of third generation (3G) with Wide Code Division Multiply Access (WCDMA) air interface. WCDMA is used to cover both Frequency Division Duplex (FDD) and Time Division Duplex (TDD) operations. UMTS integrate a wide variety of wireless multimedia services with high data transmission rates, capable of providing much more than basic voice calls. As a comparison with the GSM we can found enhancement in the all aspects such as; transmission speed, transmission way, data rate, error correction capabilities, channel capacity and quality of services as general. The bandwidth of a WCDMA is $5 \mathrm{MHz}$, and it is enough to provide data rates of 144 and $384 \mathrm{Kbps}$, and even $2 \mathrm{Mbps}$ in good conditions. WCDMA bandwidth can resolve more multipath than narrower bandwidths, as a result improving performance. The data modulation of all uplink channels is binary Phase Shift Keying (BPSK), and downlink channels are Quadra phase shift keying (QPSK). The WCDMA provides both error detection and error correction as channel coding scheme. Therefore the Universal Terrestrial Radio Access Network (UTRAN) employs two Forward Error Correction (FEC) schemes: convolution codes and turbo codes. Convolution coding can be used for low data rates, and turbo coding for higher rates [6].

In this paper will use the $2 \mathrm{G}$ and $3 \mathrm{G}$ coding standards as an inner codes and regarding to the regulations, the technical parameter for this inner codes will be fixed. Then will add extra outer code that will change the parameter on it to found good performance. The rest of this paper is organized as follows. In Section II, the technical design of $2 \mathrm{G}$ and $3 \mathrm{G}$ data channel codes is addressed. In Section III, the effectiveness 
of the concatenated channel to design MNCC over 2G and $3 \mathrm{G}$ is demonstrated. The system performance and simulation results are shown in Section IV. Finally, a conclusion in Section V, and future work in Section VI.

\section{GSM AND WCDMA CHANNEL CODING}

The traffic channels generally in $2 \mathrm{G}$ standard used convolution code as a main error correction technique in the UTRAN. The technical parameter that using is a coding rate equal to $1 / 2$ and constraint length equal to 5 with a generator polynomial as

$$
\begin{gathered}
G_{0}=1+D^{3}+D^{4} \\
G_{1}=1+D+D^{3}+D^{4}
\end{gathered}
$$

The 3G standard used convolution code as well, for most of the traffic channels such as Broadcast Channel $(\mathrm{BCH})$, Common Packet Channel (CPCH), Paging Channel (PCH), Dedicated Channel (DCH), Radio Access Channel (RACH), Downlink Shared Channel (DSCH), Forward Access Channel (FACH). However also used turbo code in some traffic channel that reqired high data rate witch is mainly downlink channels such as Downlink Shared Channel (DSCH) and Forward Access Channel (FACH). In this paper will focus on the convolution coding that used in $3 \mathrm{G}$ since the entire uplink channels use it as a main error correction technique. The technical paramere that used are depend on the type of channel, (see Table I) discribe the technical parameters clearly

TABLE I: WCDMA ERROR CORRECTION TECHNIQUES

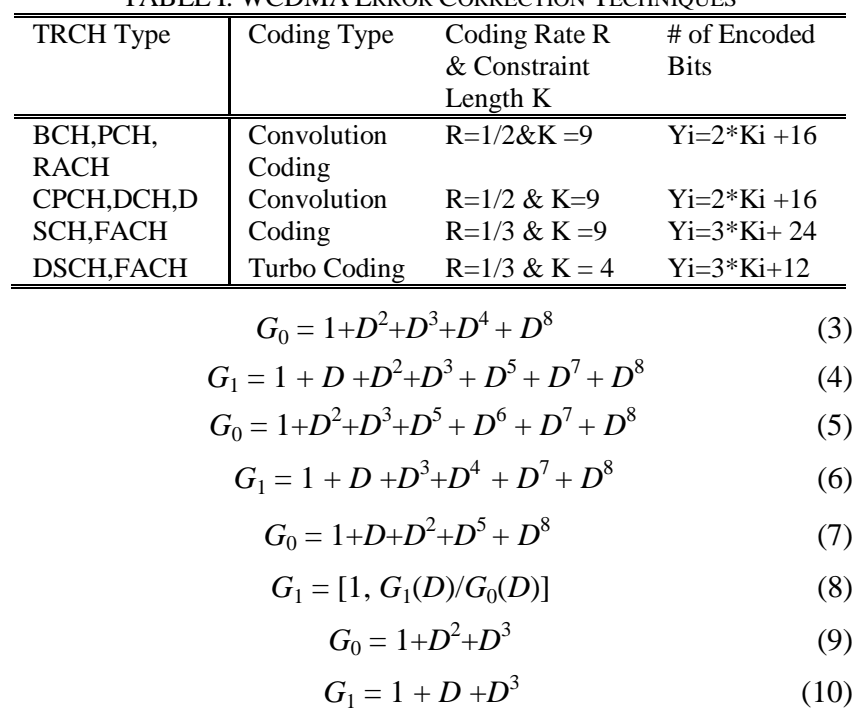

The generator polynomials that shown in (3-4) used in the convolution code with a coding rate $1 / 2$, and that shown in (5-7) used in convolution code with a coding rate $1 / 3$ and that shown in (8-10) used for the turbo code with a coding rate $1 / 3$ The Fig. 1 and Fig. 2 show the encoding structures. The decoding structure for all of them is work by convolution decoded that use soft decision Viterbi algorithm.

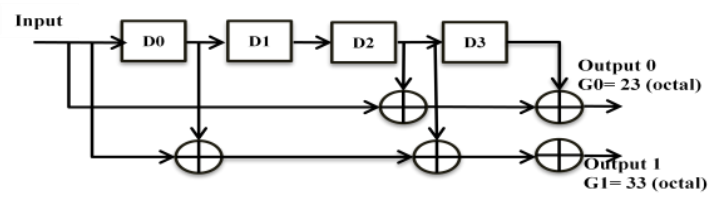

Fig. 1. Encoder structure in $2 \mathrm{G}$ cellular networks.
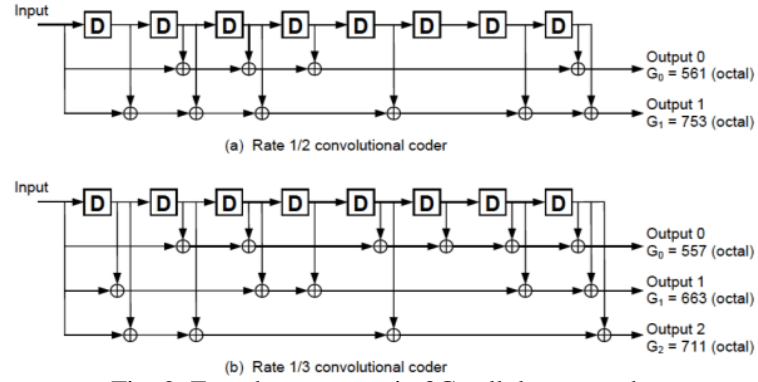

Fig. 2. Encoder structure in $3 \mathrm{G}$ cellular networks.

\section{Dependable Medical Network Channel Code SYSTEM THROUGH CELLULAR NETWORKS}

The structure of the proposal is described in the Fig. 3. The transmission rate in the GSM is $12 \mathrm{~Kb} / \mathrm{s}$ and in the WCDMA channel is $144 \mathrm{~Kb} / \mathrm{s}$, therefore will design the external outer code to provide these data rates, in order to not exceed the channel capacity of the $2 \mathrm{G}$ and $3 \mathrm{G}$ as well.

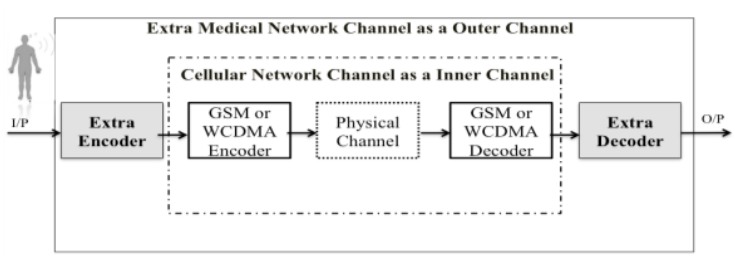

Fig. 3. The MNCC proposal structure over cellular networks.

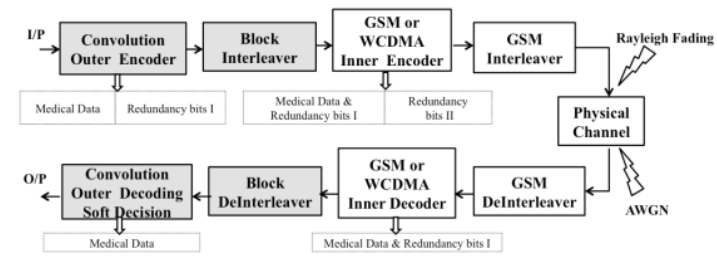

Fig. 4. The dependable medical networks structure.

\section{A. The Extra Encoder and Decoder in MNCC over $2 G$}

The extra outer encoder use convolution encoder. And extra outer interleaver uses block interleaver. The frame length $1500 \mathrm{bit} / \mathrm{s}$ is encoded with the 1/4 rate convolute code defined by the following polynomials, giving result of $6 \mathrm{~Kb} / \mathrm{s}-$ encoded bits.

$$
\begin{gathered}
G_{0}=D^{2}+D^{3}+D^{4} \\
G_{1}=1+D+D^{2}+D^{3}+D^{4} \\
G_{2}=1+D+D^{2}+D^{3}+D^{4} \\
G_{3}=1+D+D^{4} \\
G_{1}=1+D+D^{3}+D^{4}+D^{7}+D^{8}
\end{gathered}
$$

After that, the extra outer interleaver deals with a two-dimensional (encoded data/24) $\times(24)$ array. The encoded data sequence from outer encoder first written into the interleaver in the $x$ (encoded data/24) direction and then read out in the $y$ (24) direction. The inner encoder is GSM encoder that detailed in section II give output encoded data sequence equal to $12 \mathrm{~Kb} / \mathrm{s}$ same as the channel standard of GSM coder. A natural concept about a decoding algorithm for concatenated codes is to first decode the inner code and then the outer code. The inner decoder is GSM decoder that detailed in Section II. The outer de-interleaver returns the bits as the normal position. The outer decoder that we propose works Soft Decision Decoding Algorithm (SDVA); In Soft 
decision decoding, the received codeword is compared with all the possible code words by calculating the Euclidean distance and then the codeword that gives minimum Euclidean distance is selected. The whole system as in Fig. 4.

\section{B. The Extra Encoder and Decoder in MNCC over $3 G$}

The extra outer encoder use convolution encoder and extra outer interleaver use block interleaver. The technical parameters for the extra channel have two cases:

First, by use the inner code as conventional code with code rate $1 / 2$ and constraint length 9 . The frame length $24 \mathrm{Kbit} / \mathrm{s}$ is encoded with the $1 / 3$ rate convolution code with constraint length 10 defined by the polynomials equal to 1117,1365 and 1633 in octal manner, giving result of $72 \mathrm{~Kb} / \mathrm{s}$ encoded bits. After that, the extra outer interleaver deals with a two-dimensional (encoded data/5) $\times(5)$ array. The encoded data sequence from outer encoder first written into the interleaver in the $x$ (encoded data/5) direction and then read out in the $y$ (5) direction. The inner encoder is $3 \mathrm{G}$ encoder that detailed in section II give output encoded data sequence equal to $144 \mathrm{~Kb} / \mathrm{s}$ regarding to the standard of $3 \mathrm{G}$ coder.

Second, by use the inner code as a convolution code with a code rate $1 / 3$ and constraint length 9 . The frame length 16 $\mathrm{Kbit} / \mathrm{s}$ is encoded with the $1 / 3$ rate convolution code with constraint length 10 defined by the same polynomials as 1117 , 1365 and 1633 in octal manner, giving result of $48 \mathrm{~Kb} / \mathrm{s}$ encoded bits. After that, the extra outer interleaver deals with a two-dimensional (encoded data/5) $\times(5)$ array. The encoded data sequence from outer encoder first written into the interleaver in the $x$ (encoded data/5) direction and then read out in the $y(5)$ direction. The inner encoder is $3 \mathrm{G}$ encoder that we detailed in Section II give output encoded data sequence equal to $144 \mathrm{~Kb} / \mathrm{s}$ regarding to the standard of $3 \mathrm{G}$ coder. In the decoding side, the inner decoder is $3 \mathrm{G}$ decoder that detailed in Section II. The outer de-interleaver returns the bits as the normal position. At the end, the outer decoder that we propose works using SDVA.

\section{Dependable Medical Network Channel Code (MNCC) SYSTEM PERFORMANCE OVER 2G AND 3G}

\section{A. The Simulation Performance}

TABLE II: SIMULATION PARAMETERS OF MNCC

\begin{tabular}{|c|c|c|}
\hline & $\begin{array}{l}\text { 2G Simulation } \\
\text { Parameters }\end{array}$ & $\begin{array}{c}\text { 3G Simulation } \\
\text { Parameters }\end{array}$ \\
\hline "Channels & \multicolumn{2}{|c|}{ "AWGN or Rayleigh Fading } \\
\hline Modulation & GMSK & BFSK \\
\hline Receive $\mathrm{E}_{\mathrm{b}} / \mathrm{N}_{\mathrm{o}}$ & \multicolumn{2}{|c|}{$-2-18[\mathrm{~dB}]$} \\
\hline \multirow{4}{*}{ Outer Encoder } & After fix Rate $=1 / 4$ & After fix Rate $=1 / 3$ \\
\hline & After fix Constrain & After fix Constrain \\
\hline & Length $=4$ & Length $=10$ \\
\hline & $\begin{array}{l}\text { Generator module: } \\
\begin{aligned} \mathrm{G} 0 & =13, \mathrm{G} 1=15, \mathrm{G} 2 \\
& =15, \mathrm{G} 3=17\end{aligned}\end{array}$ & $\begin{array}{c}\text { Generator module: } \\
\begin{array}{c}\text { G0 = } \\
1117, \mathrm{G} 1=1365, \\
\mathrm{G} 2=1633\end{array}\end{array}$ \\
\hline Inner Encoder & $\begin{array}{l}\text { GSM Standard } \\
\text { Encoder }\end{array}$ & $\begin{array}{l}\text { WCDMA Standard } \\
\text { Encoder }\end{array}$ \\
\hline Interleaver & $\begin{array}{l}\text { Block Interleaver } \\
\text { Size } 6 \mathrm{~Kb} / \mathrm{s}\end{array}$ & $\begin{array}{l}\text { Block Interleaver Size } \\
72 \mathrm{~Kb} / \mathrm{s} \text { or } 48 \mathrm{~Kb} / \mathrm{s}\end{array}$ \\
\hline Inner Decoder & $\begin{array}{l}\text { GSM Standard } \\
\text { Decoder }\end{array}$ & $\begin{array}{l}\text { WCDMA Standard } \\
\text { Decoder }\end{array}$ \\
\hline Outer Decoder & \multicolumn{2}{|c|}{$\begin{array}{c}\text { Convolution Decoder with a Viterbi Algorithm } \\
\text { Soft Decision }\end{array}$} \\
\hline Data Bits & $\begin{array}{l}10000 \text { blocks } \\
1500 \mathrm{~b} / \mathrm{s} \text { Frame L }\end{array}$ & $\begin{array}{c}10000 \text { blocks } 24 \mathrm{~Kb} / \mathrm{s} \text { or } \\
16 \mathrm{~Kb} / \mathrm{s} \text { Frame L }\end{array}$ \\
\hline
\end{tabular}

In this part, the performance of the MNCC is evaluated using computer simulation. The parameters, which used in the simulations, are given in the Table II.

\section{B. The Simulation Results}

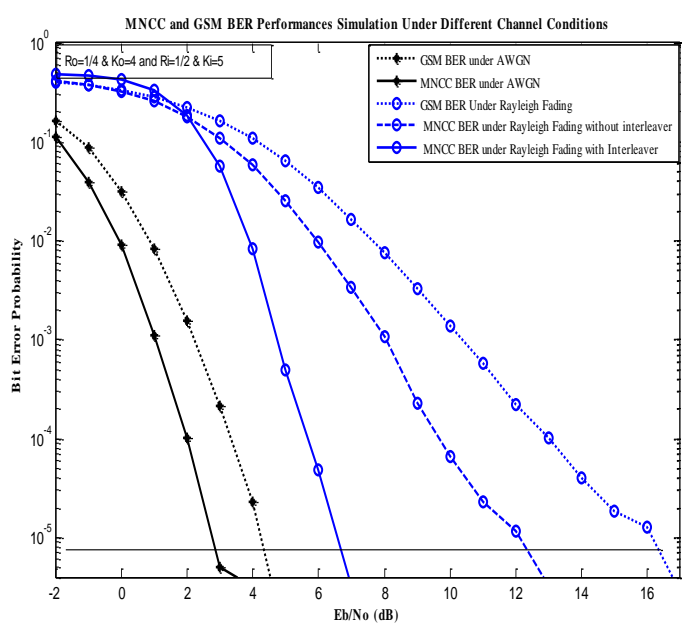

Fig. 5. The testing result under $2 \mathrm{G}$ cellular networks.

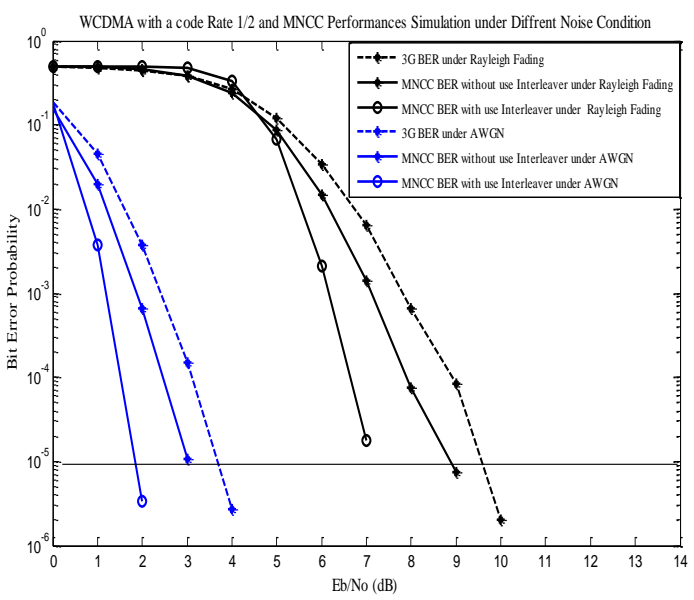

Fig. 6. The testing result under $3 \mathrm{G}$ cellular networks.

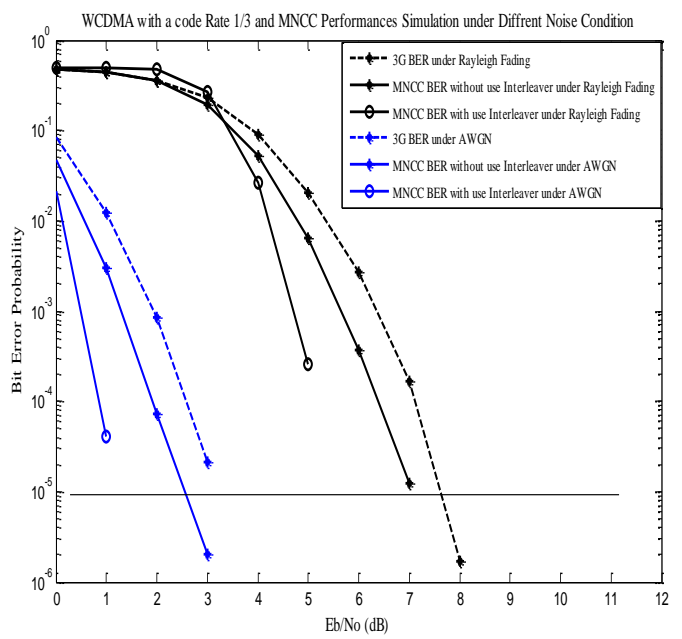

Fig. 7. The testing result under $3 \mathrm{G}$ cellular networks.

\section{CONCLUSION}

The simulation results show better performances for MNCC as compared to GSM and WCDMA and we achieved the desired BER $10^{-5}$ under AWGN, Rayleigh fading with low SNR. Generally the Proposal MNCC results as; under AWGN the Proposal system achieved BER $10^{-5}$ at $E_{b} / N_{o}=$ 
$2.2 \mathrm{~dB}$ while $2 \mathrm{G}$ achieved at $4.2 \mathrm{~dB}$ as in Fig. 5. Under Rayleigh fading the Proposal achieved BER $10^{-5}$ at $E_{b} / N_{o}=$ $7.5 \mathrm{~dB}$ while $2 \mathrm{G}$ achieved at $16 \mathrm{~dB}$ as in Fig. 5. Under AWGN the Proposal achieved BER $10^{-5}$ at $E_{b} / N_{o}=1.8 \mathrm{~dB}$ while $3 \mathrm{G}$ achieved at $3.7 \mathrm{~dB}$ as in Fig. 6. Under Rayleigh fading the Proposal achieved BER $10^{-5}$ at $E_{b} / N_{o}=7 \mathrm{~dB}$ while $3 \mathrm{G}$ achieved at $9.5 \mathrm{~dB}$ as in Fig. 6. Under AWGN the Proposal achieved BER $10^{-5}$ at $E_{b} / N_{o} \approx 1 \mathrm{~dB}$ while $3 \mathrm{G}$ achieved at about $3.5 \mathrm{~dB}$ as in fig.7. Under Rayleigh fading the Proposal achieved BER $10^{-5}$ at $E_{b} / N_{o} \approx 5.5 \mathrm{~dB}$ while $3 \mathrm{G}$ achieved at $7.8 \mathrm{~dB}$ as in Fig. 7. Then from all of that we can say this proposal rich to the physical layer issues improvement and achieving the medical transmission requirement.

\section{FUTURE WORK}

Will focusing to use iterative algorithm SOVA to decode our proposal MNCC over $3 \mathrm{G} / 4 \mathrm{G}$ and then optimize the complexity and BER to be connected to the BAN channel code.

\section{ACKNOWLEDGMENT}

I would like to express my thanks to my academic supervisor Prof. Ryuji Kohno for his help, and guidance and to all Kohno-Lab members.

\section{REFERENCES}

[1] R. H. Istepanian, B. Woodward, P. A. Balos, and S. Chen, "The comparative performance of mobile telemedical systems based on the IS-54 and GSM cellular telephone standards," J. Telemed. Telecare, vol. 5, no. 2, pp. 97-104, May 1999.

[2] B. Woodward, R. S. H. Istepanian, and C. I. Richards, "Design of a telemedicine system using a mobile telephone," IEEE Trans. Inf. Technol. Biomed., vol. 5, no. 1, pp. 13-15, 2001.

[3] S. Lin and D. J. Costello, Error Control Coding: Fundamentals and Applications, Upper Saddle River, N.J.: Pearson-Prentice Hall, 2004

[4] G. D. Forney. (1966). Concatenated Codes. Eileen Serxner Books, Inc. [Online].
http://www.abebooks.com/CONCATENATED-CODES-Forney-G-D avid-1966/1300349021/bd. [Accessed: 24-Oct-2013].

[5] E. Ahmed and R. Kohno, "Medical reliable network using concatenated channel codes through GSM network," in Proc. 2013 35th Annual International Conference of the IEEE Engineering in Medicine and Biology Society (EMBC), 2013, pp. 4755-4758.

[6] R. Steele, C. C. Lee, and P. Gould, "Capacity of GSM systems," GSM, CDMAone and $3 G$ Systems, John Wiley \& Sons, Ltd, 2001, pp. 151-204.

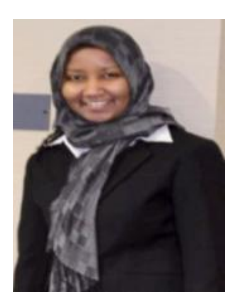

Emtithal Ahmed was born in Sudan, in 1984. She received the B.S. degree in biomedical engineering from Sudan University of Since and Technology in 2007. She received the M.S. degree from Yokohama National University, Yokohama, Japan, in 2013. She is currently working toward the Ph.D. degree in electrical and computer engineering at Yokohama National University, Yokohama, Japan. Her research interests include channel coding in wireless and mobile communications as well body area network and information coding theory and medical telemonitoring systems. She is a student member of the IEICE and IEEE.

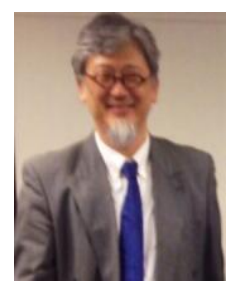

Ryuji Kohno received the $\mathrm{Ph} . \mathrm{D}$. degree from the University of Tokyo in 1984. Dr. Kohno is currently a professor of the division of physics, electrical, and computer engineering, Yokohama National University. In his career, he was the director of Advanced Telecommunications Laboratory of SONY CSL during 1998-2002 and currently the director of UWB Technology Institute of National Institute of Information and Communications Technology (NICT). In his academic activities, he was elected as a member of the board of governors of IEEE Information Theory (IT) Society in 2000 and 2003 . He has played a role of an editor of the IEEE Transactions on IT, Communications, and Intelligent Transport Systems (ITS). He is a fellow of IEICE, vice-president of Engineering Sciences Society of IEICE and has been the chairman of the IEICE Technical Committee on Spread Spectrum Technology, that on ITS, and that on Software Defined Radio (SDR). Prof. Kohno has contributed for organizing many international conferences. $\mathrm{He}$ was awarded IEICE Greatest Contribution Award and NTT DoCoMo Mobile Science Award in 1999 and 2002, respectively. He is the director of the Center for Future Medical Social Infrastructure Based on Information Communications Technology in Yokohama National University. 